\title{
How good is collaboration between maternity service providers in the Netherlands?
}

This article was published in the following Dove Press journal: Journal of Multidisciplinary Healthcare

\author{
Doug Cronie ${ }^{1,2}$ \\ Marlies Rijnders ${ }^{3}$ \\ Suze Jans ${ }^{3,4}$ \\ Corine J Verhoeven $n^{5,6}$ \\ Raymond de Vries ${ }^{7-9}$ \\ 'Department of Midwifery, OLVG \\ (West) Hospital, Amsterdam, The \\ Netherlands; ${ }^{2}$ Department of \\ Midwifery Science, Faculty of Public \\ Health and Primary Care, University \\ of Maastricht, Maastricht, The \\ Netherlands; ${ }^{3}$ Department of Child \\ Health, TNO, Leiden, The Netherlands; \\ ${ }^{4}$ Editorial Department, Dutch Journal \\ for Midwives (KNOV), Utrecht, \\ The Netherlands; ${ }^{5}$ Department of \\ Midwifery Science, AVAG, Amsterdam \\ Public Health Research Institute, \\ VU University Medical Center, \\ Amsterdam, The Netherlands; \\ ${ }^{6}$ Department of Obstetrics and \\ Gynecology, Maxima Medical Centre, \\ Veldhoven, The Netherlands; ${ }^{7}$ Faculty \\ of Midwifery Education \& Studies, \\ Zuyd University, Maastricht, The \\ Netherlands; ${ }^{8}$ CAPHRI, School for \\ Public Health and Primary Care, \\ Maastricht University, Maastricht, The \\ Netherlands; ${ }^{9}$ Center for Bioethics \\ and Social Sciences in Medicine, \\ University of Michigan Medical School, \\ Ann Arbor, MI, USA
}

Correspondence: Doug Cronie Department of Midwifery, OLVG (West) Hospital, Afdeling Verloskunde, Postbus 9243, 1006 AE Amsterdam, The Netherlands

Email d.cronie@olvg.nl
Aims: To examine the experiences of inter-professional collaboration of maternity service providers in the Netherlands and to identify potential enhancing and inhibiting factors for interprofessional collaboration within maternity care in the Netherlands.

Background: Good collaboration between health care professionals is a key element of safe, effective care, but creating a collaborative culture can be challenging. Good collaboration requires, among other things, negotiating different professional orientations and the organizational constraints of hierarchies and scheduling. Good collaboration is especially important in maternity care. In the Netherlands, suboptimal collaboration has been cited as a significant factor in maternal deaths and in adverse incidents occurring in hospitals during evenings, nights, and weekends. In spite of its importance for effective maternity care, little is known about the nature and quality of collaboration between maternity care professionals. In order to fill this gap, we examined the inter-professional collaboration within multi-disciplinary teams (MDTs) providing maternity services in the Netherlands.

Methods: Online survey of MDTs (consisting of hospital and PCMs, doctors, and carers) involved in the provision of maternity services in the Netherlands. We used a validated measure of collaboration (the Leiden Quality of Work Questionnaire) to analyze the attitudes of those involved in the provision of maternity services about multi-disciplinary collaboration in their work. We used descriptive and inferential statistics to assess differences between the groups.

Results: $40 \%$ of all respondents were not satisfied with collaboration within their MDT. Overall, mean collaboration scores (MCS) were low. We found significant differences in MCS between professional groups. Midwives - community and hospital based - were pessimistic about collaboration in future models of maternity care.

Discussion: In the Netherlands, collaboration in maternity care is less than optimal. Poor collaboration is associated with negative consequences for patient safety and quality of care. Strategies to address suboptimal collaboration exist; however, no one-size-fits-all approach is identified in the literature.

Conclusion: Suboptimal collaboration exists within the midwifery model of care in the Netherlands and the relationship between care providers is under pressure. This could affect patient safety and quality of care, according to the literature.

Précis: This paper presents an in-depth examination of the nature of, and attitudes about, collaboration between members of the MDT involved in the provision of maternity services in the Netherlands.

Keywords: interprofessional communication, communication, multi-disciplinary team, interprofessional teamwork, interprofessional collaboration survey, questionnaire hospital midwife, primary care midwife, midwifery, carer, doctor, nurse, maternity care assistant, integrated-care 


\section{Background}

Optimal collaboration between professionals involved in the provision of health care is known to be a critical element of safe, effective care, ${ }^{1-3}$ and an essential feature of effective cost management. ${ }^{4,5}$ Collaboration among the diverse professionals involved in the delivery of maternity care can be especially difficult: the challenges involved in creating a collaborative maternity care culture transcend countries and health systems. ${ }^{6,7}$

In the Netherlands, in spite of the fact that the current Dutch midwifery model is well regarded internationally, ${ }^{8,9}$ there are significant challenges to optimum collaboration. Maternity services in the Netherlands are organized into primary (community) and secondary (hospital) care, making collaboration and communication especially important. ${ }^{10}$

Midwives are the cornerstone of maternity service provision in the Netherlands, managing $>60 \%$ of all births. ${ }^{11}$ While the majority of Dutch midwives work in primary care $^{12}$ where the emphasis is on normal physiology of birth, almost one-third of all midwives now practice in a hospital setting, where the emphasis is more toward pathology and births no longer are defined as "physiological" (the Dutch term for a healthy birth).

Both groups of midwives commonly work in isolation from each other. Midwives working in primary care have developed good working relationships with other health care providers within the primary care setting. ${ }^{13}$ However, we know very little about the collaboration between hospital midwives (HMs) and other members of the multi-disciplinary maternity care team.

In the Netherlands, a midwife may be the only obstetric professional in attendance at a birth - be that birth at home or in the hospital. More than $40 \%$ of all births to women with a higher risk profile, birthing in hospital, are managed solely by HMs. ${ }^{11}$

Referral of birthing women from primary to secondary care often occurs during labor. ${ }^{14}$ This frequently involves the exchange of complex information in face-to-face communication between professionals who may or may not know each other, often by means of electronic patient-data systems that may not interface with each other. These factors present a serious challenge to the necessary collaboration between maternity service professionals.

In the Netherlands, suboptimal collaboration has been cited as a significant factor in maternal deaths and in adverse incidents occurring in Dutch hospitals during evenings, nights, and weekends. ${ }^{15,16}$ One response to these adverse outcomes has been the introduction of integrated models of care ${ }^{17}$ It has been suggested that these models will improve communication and result in better perinatal outcomes. ${ }^{18}$ However, there is currently no evidence to support this suggestion and there is no consensus on the best way forward. ${ }^{19}$ In fact, we know very little about the nature of, and attitudes about collaboration between maternity care professionals, making it difficult to predict the effects of new models of care.

Existing studies on the subject of collaboration in maternity care have focused, for the most part, on whether perceptions between those involved are aligned ${ }^{20}$ and what constitutes "good" collaboration. In their international literature review, Downe et $\mathrm{al}^{6}$ proposed a "toolkit" for (establishment of) effective collaboration in maternity care. van Helmond et $\mathrm{al}^{21}$ searched the existing literature for examples of "positive" collaboration and communication and found that there were several factors associated with good communication and collaboration. In addition, they found that parents' views were underrepresented in the literature. van der Lee et $\mathrm{al}^{22}$ evaluated collaboration between hospital obstetricians and primary care midwives (PCMs) in the Netherlands as a result of inter-professional education based on a model developed for that purpose in Canada. Among other things, they found that the potential for improved collaboration with secondary care providers, such as obstetricians and pediatricians was emphasized, and a need was expressed for better, respectful communication. These studies have been useful, but they tell us little about what collaboration looks like in everyday practice or how partners-in-care feel about their collaborations. The principle aim is to examine the experiences with inter-professional collaboration within multi-disciplinary teams (MDTs) involved in the provision of maternity services in the Netherlands.

The secondary objective of our study was to shed light on the current situation of inter-professional collaboration within maternity services in the Netherlands and has a wider relevance for providers of maternity services elsewhere.

\section{Methods \\ Design}

We carried out a cross-sectional survey using a self-administered, online questionnaire..$^{23}$

\section{Sample}

The study population consisted of all practicing midwives $(n=3,150)$, obstetricians, pediatricians, and residents $(n=959)$, nurses $(n=2,835)$, and maternity care assistants (MCAs; $n=9,966$ ) in the Netherlands.

For the purpose of this study, we identified four categories of maternity care providers, starting with two types of 
midwives. A "hospital midwife" is employed in a hospital in the secondary or tertiary care sector; a "primary care midwife" (PCM) is self-employed or employed by a primary care midwifery practice. In the third category "carer", we included obstetric-nurses (registered-nurses with an additional obstetric and gynecology training course), general nurses (registered nurse), and MCAs. In the Netherlands, MCAs are a distinct profession, they can be employed or self-employed, and work in different settings across primary and secondary care. ${ }^{24}$ They provide support to midwives in either setting, helping at births, and providing assistance to women with basic needs following the birth.

The last category was "doctor", consisting of obstetricians, pediatricians, and residents-in-training (most commonly to be an obstetrician, general practitioner, or specialist in tropical medicine). In addition, we offered respondents the option of "other" if their occupation did not fit into any of these categories.

We defined "multi-disciplinary team" as the collaborating professionals involved in the chain of maternity service provision in the health care region where the respondent worked.

\section{Data collection and analysis}

During the first week of March 2015, invitations were sent by email to 452 of the of 532 midwifery practices in the Netherlands that included an email address on their website. ${ }^{12}$ Practices received an email containing information about the study and a link to the survey. The survey was only available in Survey Monkey (via the link) from February 2015 until April 2015. Questions were numbered consecutively and the order of completion was fixed. However, respondents could move forward and backward and change answers while completing the questionnaire. No incentives financial or otherwise were offered and participation was entirely voluntary. Participation and return of the questionnaire was deemed to be informed consent.

An email was also sent to the head of departments in all hospitals in the Netherlands with maternity care facilities $(n=91)$ and to the national representative organization of MCAs (Kennis centrum voor kraamverzorgenden [KCK] \{knowledge center for MCAs\}) asking them to distribute the questionnaire among all maternity service professionals in their institutions.

In addition to this direct approach, we used snowball sampling. The Royal Dutch Organization of Midwives and the KCK placed a notification on their websites, asking all members to participate in this study. Members were asked to distribute the recruitment email among other colleagues after completion of the survey. After 4 weeks, a reminder email was sent to all hospitals and midwifery practices and further reminders were placed on the website and forums frequented by maternity care professionals.

There was no restriction on the number of participants per hospital, practice, or birth center. No specific measures were used to prevent multiple entries. Data were stored electronically in an encrypted database.

Collaboration was assessed using the Leiden Quality of Work Life Questionnaire for Nurses. This questionnaire is a validated instrument used to examine job satisfaction among nurses. ${ }^{22}$ The questionnaire consists of ten domains (of which workplace collaboration is one). The formulation of the questions was adjusted for maternity care professionals in consultation with the author of the questionnaire. Respondents' answers were measured on a 4-point Likert scale ranging from 1 (totally disagree) to 4 (totally agree). Negatively worded questions were reverse coded for analysis.

For the purpose of this study, we only used the domain "workplace collaboration". This domain consists of 17 factor statements, eight related to within-organization collaboration and eight related to within-MDT collaboration. The remaining factor statement from this domain relates to future collaboration within new models of care. In our study, we used the factor statements relating to within-MDT collaboration as well as the factor statement related to future collaboration within new models of care (see Figure 1).

In addition, information was collected on respondents' gender, age, profession, employment status (employed or self-employed), work environment (hospital, birth-center, or community-based), total years of experience (since qualifica-

\begin{tabular}{|l|}
\hline Mutual collaboration within my multi-disciplinary team (MDT) is good \\
\hline Within my MDT I experience other professionals more as colleagues \\
than as competitors \\
\hline Within my MDT communication is good \\
\hline Within my MDT I trust the abilities of my colleagues \\
\hline Within my MDT I feel valued by my colleagues \\
\hline Within my MDT colleagues criticize each other in an annoying way \\
\hline Within my MDT colleagues offer a helping hand if necessary \\
\hline Within my MDT colleagues give me emotional support in times of difficulty \\
\hline I trust in good cooperation within the future midwifery organisation. \\
\hline
\end{tabular}

Figure I Eight factor-statements relating to the measurement of within multidisciplinary team collaboration.

Note: Multi-disciplinary team was defined as: all professionals involved in maternityservice provision within the health care region where you work.

Abbreviation: MDT, multi-disciplinary team. 
tion), total years of experience in current job, and the number of working hours per week.

The questionnaire was piloted in a group of (ten) participants drawn from all professions represented in the sample. This resulted in the simplification of two questions and the deletion of one that had been included twice in the draft questionnaire.

\section{Statistical methods}

IBM SPSS version 22.0 (SPSS, Inc., Chicago, IL, USA) was used for data analysis. Using the Kolmogorov-Smirnov test, data were judged to be sufficiently normally distributed to allow parametric testing.

We compared four groups: HMs, PCMs, carers, and doctors. We used multiple imputation techniques to analyze missing data. In addition, we used descriptive statistics, chi-square, bivariate, and multivariable regression. We calculated a mean score for within-MDT collaboration for each group. We combined respondents' answers to each of the eight factor statements to make a mean collaboration score (MCS) for within-MDT collaboration. We then compared these scores across professions using ANOVA pairwise comparison of means. Higher scores correlate with better collaboration.

We dichotomized answers on the Likert items, using the categories "disagree" and "agree". We used $t$-tests, crosstabulation, and linear regression to assess the differences in relation to collaboration between all groups. Fisher's exact test was used when $>20 \%$ of the cells of the table had an expected count $<5$. We calculated Pearson's $r$ correlation for each factor statement within the domain against the MCS per professional group. Finally, we used multiple linear regression to look at the effect of several independent variables on collaboration. A $P$-value of 0.05 or lower was considered statistically significant.

\section{Ethical considerations}

This study is part of a larger study ${ }^{25}$ that was approved by the medical ethics committee of VU University Medical Center (reference number 2014/030). Ethical approval was not considered necessary according to Dutch legislation. ${ }^{26}$ The authors declare no conflicts of interest.

\section{Results}

We received a total of 4,073 responses for the MDT collaboration questionnaire. Of these, 151 were excluded because the professional group of the respondents was not stated. The "other" category was chosen by 64 respondents. Examination of the explanation of the respondents who chose "other" to describe their profession allowed us to add most of these responses $(n=44)$ to the category "carer". When we could not establish occupational group, responses were excluded $(n=20)$.

The remaining 3,902 responses were included for analysis. Of these, $80 \%$ contained some missing values. However, in total numbers, $<12 \%$ of values were missing. Missing data were found to be missing completely at random.

When imputed data for missing cases/values were compared with complete cases, the results did not significantly differ. Missing values resulted in slight fluctuations in the total number of respondents per professional group per question. Totals are given in each table. Response rates per profession are shown in Table 1.

Most of the respondents were female (except in the category "doctor", where $30 \%$ were male) and were not self-employed (Table 1). Notable exceptions were PCM (of whom $80 \%$ were self-employed) and doctors (of whom $36 \%$ were self-employed). Significant differences were noted in the mean ages between the professional groups with PCM being the youngest (mean 38 years, SD 9.8) and carers being the oldest (mean 47 years, SD 10.5). Significant differences were also seen in the mean total years of experience and mean years of experience in current job: PCM having the least total experience (mean 13 years, SD 8.9) and carers having the most (mean 16 years, SD 10.5). Doctors reported the least number of years of experience in their current job (mean 9 years, SD 8.1) and carers the most (mean 11 years, SD 9.4). Finally, when mean hours per week were compared with each group, there were significant differences between the groups: carers having the lowest (mean 23 hours per week, SD 9.1) and doctors, the highest (mean 47 hours per week, SD 9.9) number of working hours.

Overall, MCS were low (Table 2). The MCS for all groups was 2.99 ( $\mathrm{SD}=0.34)$. PCM scored lowest with an MCS of $2.79(\mathrm{SD}=0.37)$. MCS for $\mathrm{HM}(2.89, \mathrm{SD}=0.28)$ and doctors were also low (2.89, $\mathrm{SD}=0.33)$. Carers had the highest MCS (3.03, $\mathrm{SD}=0.32$ ).

When MCS were dichotomized - (3-4)= satisfied, $(1-2.99)=$ not satisfied $-40 \%$ of all respondents were not satisfied with the collaboration within the MDT. Levels of satisfaction varied between professional groups (31\%-68\%). MCAs were the most satisfied (68\%), followed by doctors (47\%) and HMs (44\%). PCMs were the least satisfied with collaboration $(68 \%)$.

In a pairwise comparison (independent samples $t$-test), the between-group difference of the MCS for HM and PCM 
Table I Basic characteristics of the study population

\begin{tabular}{|c|c|c|c|c|c|c|}
\hline & \multirow[t]{2}{*}{ Total n (\%) } & \multirow{2}{*}{\begin{tabular}{|l|}
$\begin{array}{l}\text { Hospital } \\
\text { midwife }\end{array}$ \\
n (\%) (SD) \\
\end{tabular}} & \multirow{2}{*}{\begin{tabular}{|l|}
$\begin{array}{l}\text { Primary care } \\
\text { midwife }\end{array}$ \\
$\mathbf{n}(\%)(\mathrm{SD})$ \\
\end{tabular}} & \multirow{2}{*}{$\begin{array}{l}\text { Doctor } \\
\text { n (\%) (SD) }\end{array}$} & \multirow{2}{*}{$\begin{array}{l}\begin{array}{l}\text { Other } \\
\text { carers }\end{array} \\
\text { n (\%) (SD) }\end{array}$} & \multirow[t]{2}{*}{$P$-value* } \\
\hline & & & & & & \\
\hline \multicolumn{7}{|l|}{ Gender } \\
\hline Male & $139(4)$ & $4(4)$ & $4(I)$ & $119(30)$ & $12(0.4)$ & \\
\hline Female & $3,5 \mid 4(96)$ & $93(96)$ & $386(99)$ & $27 I(70)$ & $2,764(99)$ & \\
\hline Mean age & & $42(9.8)$ & $38(10.5)$ & $45(9.9)$ & $47(10.5)$ & \begin{tabular}{|l|}
$\mathrm{a}, \mathrm{b}, \mathrm{c}, \mathrm{d}, \mathrm{e}, \mathrm{f}$ \\
\end{tabular} \\
\hline \multicolumn{7}{|l|}{ Employment status } \\
\hline Employed & $2,979(82)$ & $97(100)$ & $77(20)$ & $248(64)$ & $2,557(92)$ & \\
\hline Self-employed & $674(18)$ & $0(0)$ & $313(80)$ & $142(36)$ & $219(8)$ & \\
\hline \multicolumn{7}{|c|}{ Experience profession } \\
\hline Mean years & & $16(9.0)$ & I3 (8.9) & $15(9.8)$ & $16(10.5)$ & a,d,e,f \\
\hline \multicolumn{7}{|c|}{ Experience current job } \\
\hline Mean years & & $9(7.1)$ & $10(7.9)$ & $9(8.1)$ & II (9.4) & c,e,f \\
\hline \multicolumn{7}{|l|}{ Working hours } \\
\hline Mean per week & & $29(5.59)$ & $44(14.87)$ & $47(9.91)$ & $23(9.14)$ & 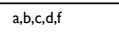 \\
\hline
\end{tabular}

Notes: $* P$-value for means = each group was compared individually to all other groups giving six possible $P$-values for each comparison. Only the $P$-values for comparisons which reach the level of significance are shown. The $P$-values are lettered as follows: ${ }^{a}=P$-value value for difference between $H M$ and $P C M$, ${ }^{b}=P$-value value for difference between HM and Drs, ${ }^{c}=P$-value value for difference between $H M$ and $M C A,{ }^{d}=P$-value value for difference between $P C M$ and $D r s, ~ e=P$-value value for difference between $P C M$ and $M C A,{ }^{f}=P$-value value for difference between Drs and MCA.

Abbreviations: Drs, doctors; HM, hospital midwife; MCA, maternity-care assistants; PCM, primary care midwife.

Table 2 Mean collaboration scores within MDT

\begin{tabular}{|l|l|l|l|l|}
\hline Professional group & $\begin{array}{l}\text { Mean within-MDT } \\
\text { collaboration (SD) }\end{array}$ & P-value* & $\begin{array}{l}\text { Not satisfied } \\
\text { n (\%) }\end{array}$ & $\begin{array}{l}\text { Satisfied } \\
\text { n (\%) }\end{array}$ \\
\hline Hospital midwives & $2.89(0.28)$ & a,c, & $53(56)$ & $4 I(44)$ \\
\hline Primary care midwives & $2.79(0.37)$ & d,e & $253(69)$ & $\mathrm{I})$ \\
\hline Doctors & $2.89(0.33)$ & d,f & I & $175(53)$ \\
\hline Carers & $3.03(0.32)$ & c,e,f & $785(32)$ & $155(47)$ \\
\hline All groups & $2.99(0.34)$ & & $\mathrm{I}, 266(40)$ & $\mathrm{I}, 63 \mathrm{I}(68)$ \\
\hline
\end{tabular}

Notes: *Each group was compared individually to all other groups giving six possible $P$-values for each comparison. Only where the $P$-value for the between-group comparison was significant is this shown. The $P$-values are lettered as follows: ${ }^{a}=P$-value value for difference between $H M$ and $P C M$, ${ }^{b}=P$-value value for difference between

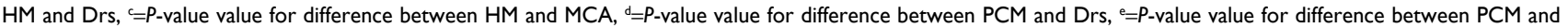
$M C A,{ }^{f}=P$-value value for difference between Drs and MCA. ${ }^{b}$ Respondents mean collaboration scores were dichotomized ( $>3=$ satisfied, $<3$ not satisfied).

Abbreviations: Drs, doctors; HM, hospital midwife; MCA, maternity-care assistants; MDT, multi-disciplinary teams; PCM, primary care midwife.

was significant $(P=0.02)$ and highly significant for the differences between HM and carers, PCM and doctors, PCM and carers, and doctors and carers $(P<0.001)$.

We found significant differences between professions on statements in the domain "within-MDT collaboration" (Table 3). More than half of the PCM (compared with less than $25 \%$ of carers) disagreed with the statement, "within my multi-disciplinary team, colleagues give me emotional support in times of difficulty" ( $52 \% \mathrm{PCM}, 23 \%$ carers, $P=0.001$ ).

One in four PCM and HM and one in five doctors (compared with one in ten of the carers) disagreed with the statement "within my multi-disciplinary team, colleagues offer a helping hand if necessary" (26\% PCM, 25\% HM, 19\% doctors, and $10 \%$ carers, $P=0.001$ ). While, $>30 \%$ of PCM (as opposed to $6 \%$ of carers) agreed with the statement "within my multi-disciplinary team, colleagues criticize each other in an annoying manner" (32\% PCM, Doctors 18\%, HM 15\%, and $6 \%$ carers, $P=0.001$ ).

Using a bivariate comparison of correlations (Pearson's $r$ ), we compared each individual factor statement within the MDT domain to the MCS for each of the professional groups. For HM, the strongest correlation was the factor statement "Within my multi-disciplinary team colleagues give me emotional support in times of difficulty" ( $r=0.687$, $P=0.001$ ). For PCM and carers, the strongest correlation was "Within my multi-disciplinary team I feel valued by my colleagues" PCM $(r=0.721, P=0.001)$ and carers $(r=0.754$, $P=0.001)$. For doctors, the strongest correlation in the domain was "Within my multi-disciplinary team I trust the abilities of my colleagues" ( $r=0.704, P=0.001)$.

When all four groups were compared with each other, both groups of midwives were less confident (than doctors 
Table 3 Between-group differences to individual factor statements from within-multi-disciplinary team collaboration domain

\begin{tabular}{|c|c|c|c|c|c|c|}
\hline & Total n (\%) & $\begin{array}{l}\text { Hospital } \\
\text { midwives n (\%) }\end{array}$ & $\begin{array}{l}\text { Primary care } \\
\text { midwives n (\%) }\end{array}$ & Doctors n (\%) & $\begin{array}{l}\text { Other care } \\
\text { providers }^{\mathrm{d}} \mathrm{n}(\%)\end{array}$ & $P$-value* \\
\hline \multicolumn{7}{|c|}{ Mutual collaboration within my multi-disciplinary team is good } \\
\hline Agree & $3,138(93)$ & $88(9 \mathrm{I})$ & $335(89)$ & $306(88)$ & $2,409(94)$ & e,f \\
\hline Disagree & $235(7)$ & $9(9)$ & $4 I(I I)$ & $43(12)$ & $142(6)$ & \\
\hline \multicolumn{7}{|c|}{ Within my multi-disciplinary team, I experience other professionals more as colleagues than competitors } \\
\hline Agree & $2,964(86)$ & $92(95)$ & $288(77)$ & $311(89)$ & $2,273(89)$ & \begin{tabular}{|l|l}
$\mathrm{a}, \mathrm{d}, \mathrm{e}$ \\
\end{tabular} \\
\hline Disagree & $409(14)$ & $5(5)$ & $88(23)$ & $38(\mathrm{II})$ & $278(\mathrm{II})$ & \\
\hline \multicolumn{7}{|c|}{ Within my multi-disciplinary team, mutual communication is good } \\
\hline Agree & $2,890(86)$ & $77(79)$ & $28 I(75)$ & $28 I(82)$ & $2,251(89)$ & c,e,f \\
\hline Disagree & $447(13)$ & $20(2 I)$ & $92(25)$ & $61(18)$ & $274(\mathrm{II})$ & \\
\hline \multicolumn{7}{|c|}{ Within my multi-disciplinary team, I trust the abilities of my colleagues } \\
\hline Agree & $3,146(93)$ & $87(90)$ & $342(92)$ & $290(85)$ & $2,427(96)$ & c,d,eff \\
\hline Disagree & $191(6)$ & $10(10)$ & $31(8)$ & $52(15)$ & $98(4)$ & \\
\hline \multicolumn{7}{|c|}{ Within my multi-disciplinary team, I feel valued by my colleagues } \\
\hline Agree & $3,024(90)$ & $86(89)$ & $282(76)$ & $312(92)$ & $2,344(94)$ & a,c,d,eff \\
\hline Disagree & $281(9)$ & $1 \mathrm{II}(\mathrm{II})$ & $89(24)$ & $27(8)$ & $154(6)$ & \\
\hline \multicolumn{7}{|c|}{ Within my multi-disciplinary team, colleagues criticize each other in an annoying way } \\
\hline Agree & $338(10)$ & $15(15)$ & $119(32)$ & $6 I(18)$ & $143(6)$ & $\mathrm{a}, \mathrm{c}, \mathrm{d}, \mathrm{e}, \mathrm{f}$ \\
\hline Disagree & $2,967(88)$ & $82(85)$ & $252(68)$ & $278(82)$ & $2,355(94)$ & \\
\hline \multicolumn{7}{|c|}{ Within my multi-disciplinary team, colleagues offer a helping hand if necessary } \\
\hline Agree & $2,838(84)$ & $71(75)$ & $27 \mid(74)$ & $267(8 \mathrm{I})$ & $2,229(90)$ & c,ef,f,d \\
\hline Disagree & $423(13)$ & $23(25)$ & $97(26)$ & $63(19)$ & $240(10)$ & \\
\hline \multicolumn{7}{|c|}{ Within my multi-disciplinary team, colleagues give me emotional support in times of difficulty } \\
\hline Agree & $2,308(68)$ & $45(48)$ & $176(48)$ & $198(60)$ & $\mathrm{I}, 889(77)$ & b,c,d,e,f \\
\hline Disagree & $943(28)$ & $49(52)$ & $192(52)$ & $132(40)$ & $570(23)$ & \\
\hline
\end{tabular}

Notes: *Each group was compared individually with all other groups giving six possible $P$-values for each factor statement. However, only where the $P$-value for the betweengroup comparison reached the level of significance is this shown. The $P$-values are lettered as follows: ${ }^{a}=P$-value value for difference between $H M$ and $P C M$, ${ }^{b}=P$-value value for difference between $H M$ and $D r s, ~ c=P$-value value for difference between $H M$ and $M C A,{ }^{d}=P$-value value for difference between $P C M$ and $D$ rs, ${ }^{e}=P$-value value for difference between PCM and MCA, ${ }^{f}=P$-value value for difference between Drs and MCA.

Abbreviations: Drs, doctors; HM, hospital midwife; MCA, maternity-care assistants; PCM, primary care midwife.

and carers) about future collaboration within new models of maternity service provision. For PCMs, this difference was significant $(P=<0.001)$, while for HMs, the difference just failed to reach the level of significance $(P=0.06)$. Carers, on the other hand were significantly more positive than the other three groups $(P=<0.001)$ regarding collaboration within new models of care.

The best-fit regression models for each professional group differed slightly (Table 4), although three factor statements were significant in each model for all professional groups. These were: "Within my multi-disciplinary team I experience other professionals more as colleagues than competitors", "Within my multi-disciplinary team colleagues criticize each other in an annoying way", and "Within my multidisciplinary team colleagues give me emotional support in times of difficulty".

\section{Discussion}

We found that overall $40 \%$ of all respondents were not satisfied with collaboration within their MDT. In addition, both groups of midwives surveyed were pessimistic over collaboration within future models of maternity service provision.

With the increased use of MDTs, especially in maternity care, optimum-collaboration is a safety issue and one of the greatest challenges of modern day health care. If collaboration is suboptimal, this may lead to medical errors. Failure to communicate effectively has been cited by the American Joint Commission for Accreditation as the third most common cause of death in US hospitals. ${ }^{27}$

Other studies report findings similar to ours. ${ }^{28,29}$ Based on these findings, our results can be broadly grouped into four groups related to collaboration: the presence of competition, trust in the abilities of colleagues, the need to feel valued (including sensitivity to criticism), and the importance of a "helping-hand" (including presence of emotional support).

Competition in health care has been found to be helpful and harmful to patient outcomes. ${ }^{30}$ Under the current system of remuneration for maternity-related care in the Netherlands, hospitals, PCMs, and home-based MCAs are reimbursed separately for their services. However, the recently published national standard for integrated care (which has been intro- 
Table 4 Linear regression model factor statements that contribute to optimal multidisciplinary collaboration per profession

\begin{tabular}{|c|c|c|c|c|c|}
\hline \multirow[t]{2}{*}{ Significant factor statement } & $\begin{array}{l}\text { Hospital } \\
\text { midwife }^{\mathrm{a}}\end{array}$ & $\begin{array}{l}\text { Primary care } \\
\text { midwife }^{\mathrm{b}}\end{array}$ & Doctor $^{c}$ & Other carer $^{d}$ & \multirow[t]{2}{*}{$P$-value* } \\
\hline & Beta $(95 \% \mathrm{Cl})$ & Beta $(95 \% \mathrm{Cl})$ & Beta $(95 \% \mathrm{Cl})$ & Beta $(95 \% \mathrm{Cl})$ & \\
\hline $\begin{array}{l}\text { Within my multi-disciplinary team, I } \\
\text { experience other professionals more as } \\
\text { colleagues than competitors }\end{array}$ & $\begin{array}{l}0.305(0.178 \text { to } \\
0.433)\end{array}$ & $\begin{array}{l}0.080(0.030 \text { to } \\
0.130)\end{array}$ & $\begin{array}{l}0.185(0.1 I I \text { to } \\
0.259)\end{array}$ & $\begin{array}{l}0.168(0.141 \text { to } \\
0.195)\end{array}$ & $\begin{array}{l}\mathrm{a}=<0.00 \mathrm{I}, \mathrm{b}=<0.02, \\
\mathrm{c}=<0.00 \mathrm{I}, \mathrm{d}=<0.00 \mathrm{I}\end{array}$ \\
\hline $\begin{array}{l}\text { Within my multi-disciplinary team, I trust the } \\
\text { abilities of my colleagues }\end{array}$ & $\begin{array}{l}0.010(-0.144 \text { to } \\
0.164)\end{array}$ & $\begin{array}{l}0.238(0.168 \text { to } \\
0.309)\end{array}$ & $\begin{array}{l}0.060(-0.029 \text { to } \\
0.149)\end{array}$ & $\begin{array}{l}0.221(0.183 \text { to } \\
0.258)\end{array}$ & $\begin{array}{l}a=0.895, b=<0.00 I, \\
c=0.183, d=<0.001\end{array}$ \\
\hline $\begin{array}{l}\text { Within my multi-disciplinary team, I feel } \\
\text { valued by my colleagues }\end{array}$ & $\begin{array}{l}0.034(-0.123 \text { to } \\
0.191)\end{array}$ & $\begin{array}{l}0.042(-0.021 \text { to } \\
0.105)\end{array}$ & $\begin{array}{l}0.169(0.063 \text { to } \\
0.275)\end{array}$ & $\begin{array}{l}0.130(0.092 \text { to } \\
0.168)\end{array}$ & $\begin{array}{l}\mathrm{a}=0.671, \mathrm{~b}=0.187, \\
\mathrm{c}=0.002, \mathrm{~d}=<0.001\end{array}$ \\
\hline $\begin{array}{l}\text { Within my multi-disciplinary team, colleagues } \\
\text { criticize each other in an annoying way }\end{array}$ & $\begin{array}{l}0.163(0.027 \text { to } \\
0.298)\end{array}$ & $\begin{array}{l}0.123(0.073 \text { to } \\
0.174)\end{array}$ & $\begin{array}{l}0.189(0.123 \text { to } \\
0.254)\end{array}$ & $\begin{array}{l}0.318(0.292 \text { to } \\
0.344)\end{array}$ & $\begin{array}{l}\mathrm{a}=0.019, \mathrm{~b}=<0.001, \\
\mathrm{c}=<0.001,{ }^{d}=<0.001\end{array}$ \\
\hline $\begin{array}{l}\text { Within my multi-disciplinary team, colleagues } \\
\text { offer a helping hand if necessary }\end{array}$ & $\begin{array}{l}0.155(0.025 \text { to } \\
0.286)\end{array}$ & $\begin{array}{l}0.048(-0.010 \text { to } \\
0.107)\end{array}$ & $\begin{array}{l}0.003(-0.084 \text { to } \\
0.091)\end{array}$ & $\begin{array}{l}0.035(0.000 \text { to } \\
0.07 I)\end{array}$ & $\begin{array}{l}a=0.20, b=0.107 \\
c=0.943, d=0.052\end{array}$ \\
\hline $\begin{array}{l}\text { Within my multi-disciplinary team, colleagues } \\
\text { give me emotional support in times of } \\
\text { difficulty }\end{array}$ & $\begin{array}{l}0.129(0.071 \text { to } \\
0.306)\end{array}$ & $\begin{array}{l}0.115(0.065 \text { to } \\
0.166)\end{array}$ & $\begin{array}{l}0.152(0.087 \text { to } \\
0.218)\end{array}$ & $\begin{array}{l}0.113(0.086 \text { to } \\
0.140)\end{array}$ & $\begin{array}{l}a=0.002, b=<0.001, \\
c=<0.001,{ }^{d}=<0.001\end{array}$ \\
\hline
\end{tabular}

Notes: *P-value for difference per profession compared with all others. ${ }^{a}=\mathrm{HM}$ compared to PCM, Dr's and carers, ${ }^{b}=$ PCM compared to HM, Dr's and carers, ${ }^{c}=$ Dr's compared to HM, PCM and carers, $\stackrel{\text { = }}{=}$ Carers compared to HM, PCM and Dr's.

duced with the expectation of improving quality of care), ${ }^{17}$ proposes an important element of integrated care, which is that caregivers will be paid from an integrated tariff, that is, a lump-sum. This policy may lead to each party receiving less money.

In our data, we see that one-in-four of the PCMs agree that they see others in the MDT more as competitors than colleagues, indicating that competition for resources is a sensitive issue. This is a potential source of mistrust between caregivers that can lead to harmful "competition" for pregnant women. ${ }^{31}$

The ability to trust colleagues was also an important issue for respondents. Trust is a fundamental component of effective collaboration. ${ }^{20,32}$ Where trust is lacking, teams are likely to be less effective, as illustrated in observational studies of obstetric emergencies. ${ }^{33,34}$

Trust is an especially important component of the Dutch model of maternity care, where birthing women are often referred during labor. ${ }^{35}$ In our findings, trust in the ability of colleagues within the MDT was strongly correlated with a higher MCS. When obstacles to trust, such as competition or role ambiguity, exist, they are likely to stand in the way of optimizing collaboration. A recent review of the role of American advanced nurse-practitioners (ANPs) in relation to others within the MDT concluded that better outcomes are achieved when ANPs have a defined role inclusive of "competencies, leadership, engagement, collaboration, and advocacy". ${ }^{36}$ Under the current model of service provision in the Netherlands, similar arrangements for HMs may assist in reducing barriers in MDT collaboration.

The need to feel valued was significant for the carers and doctors' groups in our study. Meeting this need is seen as an essential feature for effective teamwork, as widely reported among maternity service professionals in differing settings as well as in nursing teams and in teamwork situations in general. ${ }^{37,38}$ Effective teamwork - and thus effective collaboration - requires (among others) that each member of the team values the contribution of others and that criticism is undertaken from a positive perspective. Inter-professional education, structured communication (such as using situation-background-assessment-recommendation technique) and the use of no-blame incident analysis are all known to remove barriers to effective teamwork. ${ }^{17,39,40}$

Our study shows that there is room for improvement in the dynamics of multi-disciplinary maternity care teams in the Netherlands. Inter-professional education is in its infancy but is promising in its scope..$^{41}$ In addition, it has been shown that collaboration within health and social care can be improved by the introduction of national guidance and advice, ${ }^{42}$ such as the UK National Institute for Clinical Effectiveness. In the Netherlands, the recently formed College of Perinatal Care has just launched a national care standard for integrated maternity care. ${ }^{17}$ While discussion is ongoing as to how this standard can be implemented, it will nevertheless be interesting to see whether this leads to improved collaboration in the future. 
Finally, the importance of "availability of help when necessary" and "emotional support in times of difficulty" was common to all professional groups in our survey. In each of our analyses, there was a significant correlation between (the presence of) help/support and the reported score of the collaboration.

Availability of help is particularly relevant to the Dutch model of maternity care. Midwives, both in primary care and hospital settings, tend to work in isolation, large maternity units/hospitals are not common, and frequently, there will be only one obstetric professional in attendance (supported by a carer) at birth. Robust protocols exist for the provision of assistance in a homebirth setting, however, the availability of similar arrangements in hospital settings is unclear. ${ }^{43} \mathrm{In}$ spite of encouragement by the Dutch Healthcare Inspectorate (IGZ) to do so, most maternity units do not have protocols to deal with peak-pressure. ${ }^{10}$

Our findings suggest that where practitioners report that they receive help and support from colleagues, they are likely to score higher on optimal collaboration. Conversely, members of the MDT were less likely to report higher collaboration scores where there was a feeling that colleagues were seen more as competitors or that they had less trust in the abilities of their colleagues. Combined, these factors can lead to suboptimal collaboration and ineffective communication, which have been shown to compromise patient safety. ${ }^{44,45}$

Strategies to improve collaboration should be an integral feature of service provision and audit. On a positive note, more inclusive solutions are beginning to emerge. ${ }^{46}$ The introduction of perinatal audit and the formation of midwifery consortia ${ }^{47}$ may further help to improve collaboration.

Our survey is the first of its kind to report country-wide findings relating to collaboration within MDTs involved in the provision of maternity services. We used a validated tool to report the views of professionals involved.

Our survey adds to the body of knowledge regarding collaboration within maternity services and offers insight as what is important to Dutch practitioners. Our findings mirror those described in other studies underscoring their wider international relevance.

By highlighting barriers to optimal collaboration, knowledge gained from our study may be of particular value to commissioners and planners of future service provision. Our findings suggest the following key areas of concerns for practitioners: (the presence of) competition, (lack of) trust, the need to feel valued, and that the ability to help each other when necessary.

Ensuring that collaboration is optimized is a priority for practitioners and commissioners of maternity services alike.
In order to fully succeed, future models of care must reflect the concerns of service providers.

\section{Limitations}

Due to the "snowball" method used for sampling, the way in which participants in the study were recruited was different across professional groups. This fact could have introduced a selection bias. However, we are confident that any potential for bias was mitigated by our broad general appeal for participants, which appeared in the Professional Journal's and was circulated on the websites of The Professional Organizations representing our target groups.

Most of our responses contained some missing data. Although we are confident that the missing data did not adversely affect our findings, if we were to repeat the survey, we would consider redesigning the survey instrument in an attempt to minimize the chances of missing data.

In consultation with the author of the Leiden Quality of Work Questionnaire, we modified the survey instrument so that we could also include midwives and doctors. Since this did not change the tone or substance of the instrument, the modified questionnaire was not revalidated and we are confident that the (simple) modification did not affect the validity of the questionnaire.

Finally, despite our survey instrument having the facility for respondents to add additional comment for their choice of answers, very few did so. We were, therefore, unable to explore respondents' answers in-depth. A deeper understanding of the reasons for respondents' choices may improve our understanding of the challenges to optimal collaboration. We recommend further (qualitative) research into the subject.

\section{Conclusion}

Suboptimal collaboration exists within the midwifery model of care in the Netherlands and the relationship between care providers is under pressure. This could affect patient safety and quality of care, according to the literature.

Strategies to address suboptimal collaboration exist. However, no one-size-fits-all approach is apparent from the literature. Prior to the introduction of new models of care, policymakers and commissioners of maternity services should investigate which strategy is the most appropriate for each MDT in order to ensure that collaboration between care providers is optimized.

\section{Acknowledgment}

We thank Dr Margot van der Doef for giving permission to use the Leiden quality of work life questionnaire in our 
study. TNO is an independent Dutch organization for applied scientific research.

\section{Disclosure}

The authors report no conflicts of interest in this work.

\section{References}

1. McInnes S, Peters K, Bonney A, Halcomb E. An integrative review of facilitators and barriers influencing collaboration and teamwork between general practitioners and nurses working in general practice. JAdv Nurs. 2015;71(9):1973-1985.

2. Morgan S, Pullon S, McKinlay E. Observation of inter-professional collaborative practice in primary care teams: an integrative literature review. Int J Nurs Stud. 2015;52(7):1217-1230.

3. Hämel K, Vössing C. The collaboration of general practitioners and nurses in primary care: a comparative analysis of concepts and practices in Slovenia and Spain. Prim Health Care Res Dev. 2017;18(5):492-506.

4. Lewin S, Lavis JN, Oxman AD, et al. Supporting the delivery of costeffective interventions in primary health-care systems in low-income and middle-income countries: an overview of systematic reviews. Lancet. 2008;372(9642):928-939.

5. Bosch B, Mansell H. Inter-professional collaboration in health care. Lessons to be learned from competitive sports. Can Pharm J (Ott). 2015;148(4):176-179.

6. Downe S, Finlayson K, Fleming A. Creating a collaborative culture in maternity care. J Midwifery Womens Health. 2010;55(3): 250-254.

7. Ogbonnaya LU, Ogbonnaya CE, Adeoye-Sunday IM. The perception of health professions on causes of interprofessional conflict in a tertiary health institution in Abakaliki, southeast Nigeria. Niger J Med. 2007;16(2):161-168.

8. Smulders B. The Place of Birth: The Dutch Midwifery System, paper was presented at the Future Birth: The Place to be Born Conference, Australia, February 1999. Available from: https://birthinternational. com/article/midwifery/the-place-of-birth-the-dutch-midwifery-system/. Accessed November 27, 2018.

9. De Vries R, Nieuwenhuijze M, Buitendijk SE; Members of Midwifery Science Work Group. What does it take to have a strong and independent profession of midwifery? Lessons from the Netherlands. Midwifery. 2013;29(10):1122-1128.

10. Dutch Inspectorate for Healthcare (IGZ). Risks in hospital care in the evening, night and in the weekend must be better covered [Risico's ziekenhuiszorg in avond, nacht en weekend moeten beter afgedekt] (in Dutch); 2011, IGZ, Utrecht, the Netherlands. Dutch. Available from: http://www.sin-nl.org/wp-content/uploads/2015/06/201109-Rapport-ANW_tcm294-308091.pdf. Accessed November 21, 2018.

11. Cronie D, Rijnders M, Buitendijk S. Diversity in the scope and practice of hospital-based midwives in the Netherlands. J Midwifery Womens Health. 2012;57(5):469-475.

12. Kenens R, Battenburg R, Kasteleijn A. Numbers from the registration of midwives [Cifers uit de registratie van verloskundigen]. Nivel, Utrecht. 2016. Dutch. Available from: https://www.nivel.nl/sites/default/files/ cijfers-uit-de-registratie-van-verloskundigen-peiling-jan-2016.pdf. Accessed November 21, 2018.

13. Manniën J, Klomp T, Weigers T, et al. Evaluation of primary care midwifery in the Netherlands: design and rationale of a dynamic cohort study (DELIVER). BMC Health Serv Res. 2012;12:69.

14. Offerhaus PM, Hukkelhoven CW, de Jonge A, van der Pal-de Bruin KM, Scheepers PL, Lagro-Janssen AL. Persisting rise in referrals during labor in primary midwife-led care in the Netherlands. Birth. 2013;40(3):192-201.

15. Schutte JM, Steegers EA, Schuitemaker NW, et al. Rise in maternal mortality in the Netherlands. BJOG. 2010;117(4):399-406.
16. Kramer J, Cronie D, Smit M, Schraagen J. Evaluation of Midwifery Protocols in the Netherlands; an analysis of task and responsibilitiy agreements among hospital maternity service providers [Verloskundige Protocol Evaluatie Nederland; een analyse van taak en verantwoordelijkheidsafspraken van tweedelijns zorgverleners] TNO-report 031.20452;13 (in Dutch); October 2010.

17. College Perinatal Care (CPZ). Care standard "integrated perinatal care" [Zorgstadaard "Integrale geboortezorg" Versie een] Version 1.0 College Perinatal Care (in Dutch); 2015. Utrecht, the Netherlands.

18. van der Velden J, Pultrum J, Becker Hoff J. et al. A good beginning: safe care around pregnancy and birth. Advice of the steering group pregnancy and birth. [een goed begin: veilig zorg rondom zwangerschap en geboorte. Advies van stuurgroep] Government Law (in Dutch); 2009. Available from: http://www.rijksoverheid.nl/enzwangerschap-en/x-cz2978049b.pdf/. Accessed June 26, 2016.

19. Perdok H, Jans S, Verhoeven C, et al. Opinions of maternity care professionals and other stakeholders about integration of maternity care: a qualitative study in the Netherlands. BMC Pregnancy Childbirth. 2016;16(1):188.

20. Romijn A, Teunissen PW, de Bruijne MC, Wagner C, de Groot CJM. Interprofessional collaboration among care professionals in obstetrical care: are perceptions aligned? BMJ Qual Saf. 2018;27(4): 279-286.

21. van Helmond I, Korstjens I, Mesman J, et al. What makes for good collaboration and communication in maternity care? A scoping study. Int J Childbirth. 2010;5:210-223.

22. van der Lee N, Driessen E, Scheele F. How the past influences interprofessional collaboration between obstetricians and midwives in the Netherlands: findings from a secondary analysis. $J$ Interprof Care. 2016;30(1):71-76.

23. van der Doef M, Maes S. The leiden quality of work questionnaire: its construction, factor structure, and psychometric qualities. Psychol Rep. 1999;85(3):954-962.

24. van Teijlingen ER. The profession of maternity home care assistant and its significance for the Dutch midwifery profession. Int J Nurs Stud. 1990;27(4):355-366.

25. Verhoeven $C$ [homepage on the Internet]. INCAS-2 (INtegrated CAre System) a study into integrated care in the Netherlands; 2017. Available from: http://www.incas2.nl/. Accessed March 23, 2017.

26. Dutch Central Committee for Research Involving Human Subjects, CCMO; 2017. Available from: https://english.ccmo.nl./investigators/ legal-framework-for-medical-scientific-research/your-research-is-itsubject-to-the-wmo-or-not. Accessed November 21, 2018.

27. Joint Commission on Accreditation of Healthcare Organizations (JCAHO). Sentinel event statistics released for 2014 Joint Commission on Accreditation of Healthcare Organizations; 2015. Available from: https://www.jointcommission.org/assets/1/23/jconline_April_29_15. pdf. Accessed April 2018.

28. Henneman EA, Lee JL, Cohen JI. Collaboration: a concept analysis. J Adv Nurs. 1995;21(1):103-109.

29. Reiger KM, Lane KL. Working together: collaboration between midwives and doctors in public hospitals. Aust Health Rev. 2009;33(2): 315-324.

30. Barros P, Werner B, Brouwer F, Thomson S, Varkevisser M. Competition among health care providers: helpful or harmful? Eur. J Health Econ. 2015;2016(17):229-233.

31. Royal Dutch Organisation of Midwives (KNOV). Standpoint Integrated Funding [Standpunt integralebekostigen] (in Dutch). Utrecht: KNOV; 2013. Available from: https:/www.knov.nl/werk-en-organisatie/tekstpagina/128-2/integrale-bekostiging/hoofdstuk/1107/standpunt-knovintegrale-bekostiging/. Accessed November 21, 2018.

32. Costa A, Roe R, Taillieu T. Trust within teams: the relation with performance effectiveness. Eur J Work Organ Psychol. 2010;10(3):225-244.

33. Siassakos D, Crofts J, Winter C, Weiner C, Draycott T. The active components of effective training in obstetric emergencies. BJOG. 2009;116(8): $1028-32$.

34. Madden E, Sinclair M, Wright M. The Royal College of Midwives. Teamwork in Obstetric Emergencies. Evidence Based Midwifery. 2011. 
35. Blix E, Kumle M, Kjærgaard H, Øian P, Lindgren HE. Transfer to hospital in planned home births: a systematic review. BMC Pregnancy Childbirth. 2014;14:179.

36. McIntosh D, Startsman LF, Perraud S. Mini review of integrated care and implications for advanced practice nurse role. Open Nurs $J$. 2016;10:78-89.

37. Baker DP, Day R, Salas E. Teamwork as an essential component of highreliability organizations. Health Serv Res. 2006;41(4 Pt 2):1576-1598.

38. Kalisch BJ, Weaver SJ, Salas E. What does nursing teamwork look like? A qualitative study. J Nurs Care Qual. 2009;24(4):298-307.

39. Avery MD, Montgomery O, Brandl-Salutz E. Essential components of successful collaborative maternity care models: the ACOG-ACNM project. Obstet Gynecol Clin North Am. 2012;39(3):423-434.

40. van der Lee N. Tailoring CanMEDS for Training in Obstetrics and Gynaecology in the Netherlands. Amsterdam; Vu University, 2014.

41. Koelewijn J. Does the SBAR-method improve handover and collaboration? Midwives Journal (TvV). 2014. Dutch. Available from: https://tvv. knov.nl/artikelen/detail/203/verbetert-de-sbar-methode-de-overdrachten-de-samenwerking. Accessed November 21, 2018.

42. Sakala C, Corry M. Evidence-Based Maternity Care: What It Is and What It Can Achieve. New York, USA: Millbank Memorial Fund; 2008.
43. Cronie DJ, Rijnders M, de Vries R, Buitendijk S. Making evidence available for hospital-based midwives: a systematic examination of the content and methodological quality of three protocols used in maternity hospitals in the Netherlands. Int $J$ Childbirth. 2014;4(4):208-218.

44. Lyndon A, Zlatnik M, Watcher R. Effective physician-nurse communication. a patient safety essential for labor \& delivery. Am J Obstet Gynecol. 2011;205(2):91-96.

45. Martijn L, Jacobs A, Amelink-Verburg M, Wentzel R, Buitendijk S, Wensing M. Adverse outcomes in maternity care for women with a low risk profile in the Netherlands: a case series analysis. BMC Pregnancy Childbirth. 2013;13(13):219.

46. Posthumus AG, Schölmerich VL, Waelput AJ, et al. Bridging between professionals in perinatal care: towards shared care in the Netherlands. Matern Child Health J. 2013;17(10):1981-1989.

47. van Diem MT, Bergman KA, Bouman K, et al. Perinatale audit NoordNederland: de eerste 2 jaar. [Perinatal audit in the North of the Netherlands: the first 2 years]. Dutch Med J [Nederlands Tijdschrift voor Geneeskunde]. 2011;155(18):A2892. Dutch.
Journal of Multidisciplinary Healthcare

\section{Publish your work in this journal}

The Journal of Multidisciplinary Healthcare is an international, peerreviewed open-access journal that aims to represent and publish research in healthcare areas delivered by practitioners of different disciplines. This includes studies and reviews conducted by multidisciplinary teams as well as research which evaluates the results or conduct of such teams or health
Dovepress

care processes in general. The journal covers a very wide range of areas and welcomes submissions from practitioners at all levels, from all over the world. The manuscript management system is completely online and includes a very quick and fair peer-review system. Visit http://www.dovepress.com/ testimonials.php to read real quotes from published authors.

Submit your manuscript here: https://www.dovepress.com/journal-of-multidisciplinary-healthcare-journal 